\title{
Teleophthalmology and Artificial Intelligence As Game Changers in Ophthalmic Care After the COVID-19 Pandemic
}

\author{
Anna Nikolaidou ${ }^{1}$, Konstantinos T. Tsaousis ${ }^{2}$ \\ 1. Ophthalmology, Aristotle University of Thessaloniki, Thessaloniki, GRC 2. Ophthalmology, Volos General Hospital, \\ Volos, GRC
}

Corresponding author: Konstantinos T. Tsaousis, konstantinos.tsaousis@gmail.com

\begin{abstract}
The current COVID-19 pandemic has boosted a sudden demand for telemedicine due to quarantine and travel restrictions. The exponential increase in the use of telemedicine is expected to affect ophthalmology drastically. The aim of this review is to discuss the utility, effectiveness and challenges of teleophthalmological new tools for eyecare delivery as well as its implementation and possible facilitation with artificial intelligence. We used the terms: "teleophthalmology," "telemedicine and COVID-19," "retinal diseases and telemedicine," "virtual ophthalmology," "cost effectiveness of teleophthalmology," "pediatric teleophthalmology," "Artificial intelligence and ophthalmology," "Glaucoma and teleophthalmology” and "teleophthalmology limitations" in the database of PubMed and selected the articles being published in the course of 2015-2020. After the initial search, 321 articles returned as relevant. A meticulous screening followed and eventually 103 published manuscripts were included and used as our references. Emerging in the market, teleophthalmology is showing great potential for the future of ophthalmological care, benefiting both patients and ophthalmologists in times of pandemics. The spectrum of eye diseases that could benefit from teleophthalmology is wide, including mostly retinal diseases such as diabetic retinopathy, retinopathy of prematurity, age-related macular degeneration but also glaucoma and anterior segment conditions. Simultaneously, artificial intelligence provides ways of implementing teleophthalmology easier and with better outcomes, contributing as significant changing factors for ophthalmology practice after the COVID19 pandemic.
\end{abstract}

Review began 06/23/2021 Review ended 07/13/2021 Published 07/14/2021

๑) Copyright 2021

Nikolaidou et al. This is an open access article distributed under the terms of the Creative Commons Attribution License CC-BY 4.0., which permits unrestricted use, distribution, and reproduction in any medium, provided the original author and source are credited.
Categories: Ophthalmology

Keywords: teleophthalmology, artificial intelligence, covid-19, virtual clinics, machine learning

\section{Introduction And Background}

As the SARS-COVID-19 pandemic arose in 2019, so did the implementation of telemedicine in a wide range of medical fields, among which, ophthalmology may be one of the most benefited ones [1]. "Telemedicine" means medicine from a distance, deriving from the Greek prefix “tele," which means "from afar.” A subsection of telemedicine, teleophthalmology, involves telecommunication tools such as smartphones, powerful hardware, advanced software, wireless devices, and remote video tools but also applications of artificial intelligence (AI) [2-4]. There are two methods of exploiting teleophthalmology: synchronous and asynchronous, as shown in Figure 1. Between the two, the noticeable difference is the time required for diagnosis and consultation, while both result in the recording of data. Providing healthcare from a distance, teleophthalmology has in recent years been in the spotlight of modern medicine, which was further triggered by the pandemic [5]. 


\section{Cureus}

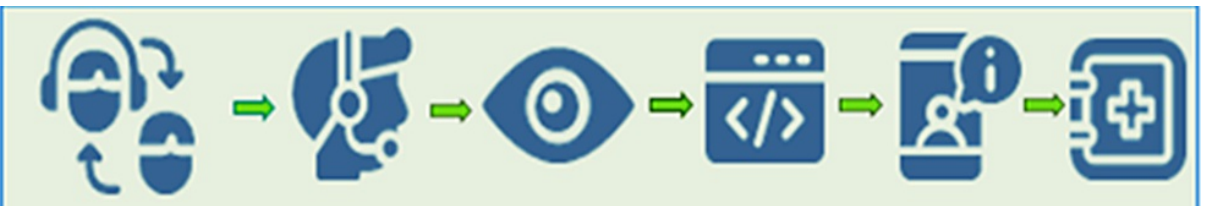

synchronous method: video calling, eye photography, immediate counseling and diagnosis, recording

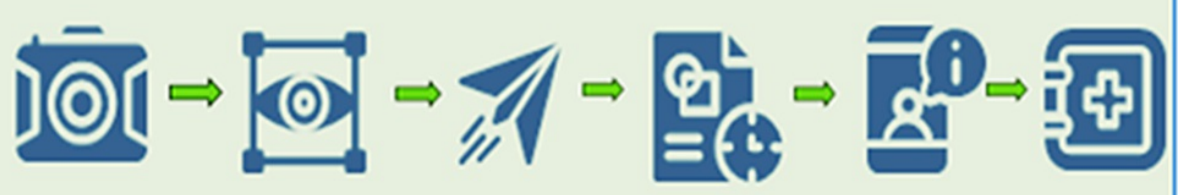

asynchronous method: photography and file upload, consulting and delayed diagnosis, recording FIGURE 1: Synchronous and asynchronous methods of
teleophthalmology.

In ophthalmology, a medical field that follows and benefits from technological advances, the evaluation and monitoring of patients remotely proves to be very helpful for both patients and health professionals. Teleophthalmology can benefit patients in a variety of ways by eliminating distances [6], reducing waiting times and intervals between visits and minimizing the risk of nosocomial infections for vulnerable groups. Thus, decongesting ophthalmology clinics also gives the opportunity to healthcare providers for better patient screening and more complete data recording [7]. Among others, diabetic retinopathy (DR), agerelated macular degeneration, glaucoma, cataract and premature retinopathy are diagnosed early and monitored through devices and applications of AI [8,9].

Teleophthalmological practices can also be of great importance for circumstances like natural disasters, infectious disease outbreaks and, in general, whenever, and wherever healthcare is limited or inaccessible like in rural areas, remote villages and poorly resourced regions [10-14]. It has also been reported that even soldiers can benefit from teleophthalmology, avoiding unnecessary evacuation from warzone according to Cocozza et al. [15].

However, concerns arise about the immediacy of the procedure and the quality of the doctor-patient relationship that is likely to deteriorate. The application of teleophthalmology requires training in the use of devices by health professionals, which can be a burden for its wide use. Furthermore, patient accessibility is questioned especially for elderly patients who are less familiar with the technology. These challenges can trigger the effective use of teleophthalmology, which profits the established lifelong ophthalmological examination, but it cannot replace it even if its sensitivity and specificity prove to be of high value [16,17].

Undoubtedly, the SARS-COVID-19 pandemic constitutes a period that ophthalmological consultation that can be challenging due to the short distance between the ophthalmologist and the patient. Therefore, patients with chronic eye diseases that need monitoring and routine screening and patients that require follow-up visits are examples of cases that can avoid the hospital environment that comes with high viral exposure and use telemedicine [18]. Additionally, teleconsultation can be useful for the health professionals that are quarantined due to potential exposure to the virus, offering the chance to keep a close eye on their patients from a distance [19].

\section{Review}

\section{Diseases - implementation}

1. Retinal Disorders

Image-based investigations are a crucial part of both retinal disorders and teleophthalmology implementations. Thus, the field of the retina is probably the one that teleophthalmology has benefited the most for both screening and follow up. For adult patients, DR and for pediatric patients, retinopathy of prematurity (ROP) are at the heart of teleophthalmological advances and applications.

The growing need for evaluation of retinal diseases is a key sector that teleophthalmology can thrive in, using synchronous and asynchronous methods for consultation. De Fauw et al. showcased that AI has the potential to be very useful for retinal diseases. An enormous number of images can be generated and 
processed with deep learning techniques [20]. Advanced fundus imaging techniques and optical coherence tomography (OCT) imaging have enabled the growth of retinal teleophthalmology systems, where remote imaging containing important clinical information is centrally interpreted [21]. Careful and proper integration of the retinal telemedicine programs in the current clinical practice will be significant for their proper use and the creation of virtual clinics in the medical retina may change the eye care delivery soon, as displayed in El-Khayat et al. [22,23].

Recent studies have shown that teleophthalmology can be an accurate and sensitive tool for screening in DR and is promising to be a universal newborn screening tool [24,25]. Additionally, Kozak et al. [26] highlighted in a prospective, interventional cohort study that remotely a retina specialist can also implement interventions such as telephotocoagulation for diabetic macular edema.

(a) Diabetic retinopathy (DR): It constitutes one of the most important complications of diabetes mellitus and is the leading cause of visual disability for DM patients. It is estimated that 486 million people worldwide have DM and almost $1 / 3$ suffers from DR while the visual-threatening cases are increasing according to Yau et al. [27,28]. In this setting, teleophthalmological tools are essential for screening, management and follow up of DR. Retinal imaging is achieved with portable screening devices such as mobile imaging units, smartphone-based retinal photography and applications of AI providing high sensitivity and specificity $[4,29,30]$. Images are either evaluated by a remote ophthalmologist or automatically analyzed by a deep learning algorithm [31]. Also, diabetic macular thickening can be predicted through color fundus photographs from deep learning models demonstrated by Arcadu et al. enhancing the efficiency of DM diagnosis [32].

Screening with teleophthalmological means can have a significant impact on reducing vision loss caused by DR. Nationwide-based systems but also community-based programs are proving to boost the screening rates and result in avoiding visual complications around the world [33]. Tozel et al. completed a review of large DR telemedicine screening programs for DR across the globe and demonstrated that a high level of clinical accuracy and easier patient access are accomplished [34]. Unnecessary referral minimizing and cost-saving are also reported in recent studies for both rural population and urban community teleophthalmology services $[35,36]$.

In several city-specific studies, such as Vujosevic et al. in Padova and Invernizzi et al. in Milan, (singlecentre) teleophthalmology screening for DR proved to be convenient and well-received by patients and resulted in avoiding unnecessary referrals and less costs than in the standard slit-lamp examination [37, 38]. In the United Kingdom, the nationwide teleophthalmological screening program has been described as a great opportunity for DR patients that are least likely to attend a screening test [39]. Likewise, in Canada, both urban and rural programs of teleophthalmology have benefited geographically disparate populations and urged the guidance of healthcare delivery across the country [40]. Furthermore, in Zimbabwe Retinopathy Telemedicine Project (ZRTP), teleophthalmological screening gave an opportunity to limitedaccess patients to not only manage DR but also educate themselves on DR [41].

Widespread implementation of teleophthalmology for DR has been in the spotlight also in the USA, as the growing number of people having diabetes mellitus requires many specialists and screening visits. The teleophthalmological approach can annihilate distances and provide a wide range of specialists for consultation or automated consultation. However, as with every new technology, there are barriers to overcome, economic and utility and familiarity as Liu et al. explained, especially in rural settings [11,42]. In Chile, the use of telemedicine resulted in a boost/surge for screening coverage of DR patients and helped to overcome the lack of specialists as seen in Avendano-Veloso et al. [43].

As for imaging methods, Silva et al. compared DR identification between non-mydriatic ultrawide field (UWF) imaging and non-mydriatic multi-field fundus photography (NMFP) in a large ocular telehealth program and demonstrated significant benefits of the UWF imaging method [44]. The "reading" of the retinal images can be done by many health professionals like optometrists, general ophthalmologists and retinal specialists without significant variability, but with the training needed to improve consensus on image grading as Liu et al. highlighted [45]. As for the imaging, that can also be done by nonphysicians [46]. Pharmacy-based teleophthalmology was suggested by Coronado et al. in a study conducted in semi-urban areas of Ontario, Canada, choosing pharmacies as a strategic place that DM patients visit to acquire medications and proved to be effective on wide screening but not cost-effective compared to slit-lamp examination [47].

Follow-up care after the screening of DR is required to have effective screening and as demonstrated by Boucher et al. in a cross-sectional retrospective descriptive study, a high number of compliances was achieved in an urban community-based DR teleophthalmology project with a $91.9 \%$ compliance rate. Also, a high patient satisfaction score was achieved, which is also required for effective screening [48].

(b) Age-related macular degeneration (AMD): It is the main cause of visual impairment and blindness in Europe and the USA [49,50]. Almost 67 million people across Europe suffer from AMD and while the life expectancy is increasing, and by 2050 this number is expected to reach 77 million [49]. The lack of a well- 
defined and specific high-risk population for AMD is a limitation for the successful implementation of teleophthalmology for AMD. Teleophthalmological means AMD may require the extension of the remote screening tool kit to a network-connected fundus camera that includes technologies such as OCT and possibly OCT angiography.

Brady et al. reviewed the state-of-the-art telemedicine programs for AMD: some added screening for AMD in the already-existing DR telemedicine programs with positive results while others found AMD telemedicine programs to be ineffective economically [51]. Kawaguchi et al. in 2018 demonstrated that teleophthalmological programs for screening AMD are equally effective as the traditional slit-lamp examinations while having a statistically significant higher possibility of patient participation [52]. The "Muranga teleophthalmology study" in Kenya showcased a fair agreement of slit-lamp examination and web-based teleophthalmology assessment in both rural and urban settings. Additionally, $99 \%$ of patients were satisfied with the great majority preferring teleophthalmology than slit-lamp examination [53]. A randomized clinical trial in Canada proposed the benefit of teleophthalmology for health professionals working in such programs to expand their knowledge in retinal services under the teleophthalmic guidance of retinal specialists and highlighted the need for such programs in remote areas so that timely diagnosis of AMD and its monitoring are accessible for everyone [54].

The model of "virtual" follow-up appointments proved to be greatly helpful to contend with capacity issues as resulted in a single-center study by Tsaousis et al.: decreasing the time between appointments, improving the number of injections/patients/years, and most drastically boosting mean visual acuity (VA) improvement $>15$ letters [55]. Self-monitoring with a mobile application for AMD, proposed by Schmid et al., showed reasonable accuracy to detect wet AMD and classify dry vs. wet AMD. This kind of application also provides valuable data for patients monitoring through the years that are crucial for diagnosis and management [56].

(c) Retinopathy of prematurity (ROP): It constitutes a leading cause of preventable childhood blindness or visual impairment worldwide [57]. This proliferative disorder affects premature infants, usually of low birth weight. Inadequate screening is considered to be the cause of the high prevalence of ROP instead of the continuous advances in treatment like anti-vascular endothelial growth factor (anti-VEGF) agents and photocoagulation [58]. Teleophthalmology with fundus photography and remote grading by experienced ophthalmologists can drastically change the percentage of access to ophthalmic care for patients, especially in poorly resourced communities. When compared to binocular indirect ophthalmoscopy examination, telemedicine grading of dilated fundus imaging, no statistically significant difference in the sensitivity was detected in a multicenter study conducted by Biten et al. Both methods showed high inter-examiner variability [59]. Smartphone-based fundus photography systems are suggested by researchers across the world as the "need of the hour" for ROP proving to be of high feasibility and accuracy [60], cost-effective and user-friendly [61]. With a rising incidence of ROP and a limited number of adequately trained ophthalmologists, the need for an easy-using, fast and effective tool for screening is urgent [62,63]. Offering high image quality and affordability of imaging systems, telemedicine constitutes a valuable solution for vulnerable pediatric patients [64].

AI could not be missing from teleophthalmology instruments for ROP. Brown et al. proposed an algorithm using deep convolutional neural networks (CNN) to diagnose plus disease in ROP with comparable or better accuracy by human experts. Infants at risk of ROP in resource-limited or remote areas can benefit from such algorithms, using technology to prevent, detect and manage ROP [65]. In a recent review, Scruggs et al. highlighted the advantage of AI in the ROP diagnosis offering objectivity and improved accuracy (as a paradigm-shifting strategy to improve efficiency) [66]. Hu et al. proposed deep CNN are to identify the presence and severity of ROP disease per-examination using an automated analysis strategy. The model was proved to be effective with the only limitation being the falsely recognized reflection of light, as the ridge of ROP, resulting in an incorrect prediction outcome [67].

\section{Glaucoma}

Glaucoma constitutes one of the leading causes of irreversible blindness worldwide affecting mostly women and Asians [68]. Screening, diagnostic consultation and long-term treatment monitoring of glaucoma overcome the barriers of distance when using telemedicine [69]. Aiming to high-quality, cost-effective care to achieve the appropriate balance between clinical, economic and humanistic objectives, telemedicine is needed due to insufficient drug or intervention methods as presented in Delgrado et al. [70]. In LVPEI Glaucoma Epidemiology and Molecular Genetic Study, a rural population-based study, teleophthalmoscopic grading of peripheral anterior chamber depth with Van Herick $(\mathrm{vH})$ technique showed that combined vH grading and ocular biometry increased the predictability of a gonioscopically occludable angle [71].

A pilot program in the USA using e-health to monitor low-risk glaucoma suspects achieved high patient follow-up compliance, proving telemedicine to be promising for glaucoma suspects [72]. Another study in the USA evaluated the accordance between teleglaucoma consultation and in-person assessment for glaucoma progression. The results showed high similarity between the two methods, suggesting teleophthalmology as an auxiliary tool to decongest clinics but with limitations due to a lack of absolute agreement [73]. 
Diagnostic accuracy, screening effectiveness and evaluation of progression risk can be facilitated by AI technologies using deep learning algorithms as presented by Mursch-Edlmayr et al. Specificity and sensitivity for OCT imaging, fundus photography and visual field (VF) testing are increased when using AI. For glaucoma, AI can predict progression earlier than conventional methods, but incorporation into the health system is needed so that it makes the most out of the benefits of AI [74]. AI can act as a form of teleophthalmological care for glaucoma, collecting data from devices that the patients use at home such as self-monitoring of intraocular pressure tonometry [75], smartphone-based head-mounted perimeter for detection of VF defects [76], ophthalmoscopic applications for smartphones and processing this data to result in a diagnosis or progression risk percentage [77]. Effective triage of the patients and facilitation of glaucoma clinical practice constitute fields that telemedicine is expected to thrive when Glaucoma AI algorithms meet regulatory approval [78].

\section{Pediatric Eye Disease}

The use of telemedicine can help to reduce delays in referrals and developing new specialized centers with teleophthalmology services is thought to be the future for pediatric ophthalmology in which timely referral can avoid vision permanent loss [79]. VA was evaluated with a phone application called GoCheckKids and the results showed a modest correlation of VA compared to a regular clinic protocol showing potential for future VA evaluation at home [80]. Video-recorded screening for assessing children was proposed by Sabri et al. in a study for pediatric amblyopia and eye disease that trained non-eye-care professionals can record adequate videos for a remote ophthalmologist to consider when there is no direct access for consultation [81].

As for postoperative care, teleophthalmology can also contribute to virtual visits for pediatric patients, which can be more pleasant for the children because of decreased waiting times with quality care maintained [82].

AI for pediatric eye disease is yet to be developed as for the adults, but significant advances have been reported as "classification of pediatric cataracts, prediction of postoperative complications following cataract surgery, detection of strabismus and refractive error, prediction of future high myopia, and diagnosis of reading disability." Applications of AI are expected to pave the way for optimal clinical care for children with early disease detection and grading but most essentially widely accessed care [83].

\section{Anterior Segment Conditions}

Keratoconus, infectious keratitis, refractive surgery, corneal transplant, adult and pediatric cataracts, angleclosure glaucoma, iris tumors, post-refractive surgery keratitis and post-cataract surgery endophthalmitis constitute anterior segment conditions that could benefit from teleophthalmology and its AI applications but the efficiency of this is yet to be widely investigated [84-86].

Smartphone-assisted anterior segment imaging was performed in Iran with 10 and 90 Diopter lenses commercially available in ophthalmic settings, proving to be a useful alternative when a slit lamp is not available or the patient is bedridden or hospitalized [87]. In rural India in the EyeSmart study-I, teleophthalmology has been presented as a promising tool for the management and diagnosis of anterior segment diseases but also for the lid and adnexal pathologies. A pyramidal model was used for eye care delivery, in which vision technicians photographed patients' eyes and then teleconsultation was conducted if needed. This model with the use of EyeSmart application proved to overcome the barriers of distance, time and costs, for mostly lens-related (38.3\%) followed by ocular surface pathologies (30.2\%), lid and adnexa-related pathologies (8.6\%), and corneal pathologies [86].

The application of AI to the diagnosis and management of cataracts was recently extensively analyzed by Ting et al. [88], but its applications in teleophthalmology are still not extensively researched. Integration of AI technology with telemedicine healthcare systems for anterior segment diseases requires large sample studies to ensure accuracy. Wu et al. presented a deep learning algorithm, ResNet, for the assessment and diagnosis of adult and pediatric cataracts of varying severities in a real-world setting. This algorithm, when combined with teleophthalmological tools (ocular surface images taken by cell phones, applications for clinical history and VA) and referral to community-based healthcare facilities and the tertiary eye hospitals, showed optimal diagnostic performance in grading (AUC > 0.99) [89].

\section{Emergencies}

Ocular fundus photography with teleophthalmological means can bring patients to medical attention at an earlier stage of disease, such as intracranial hypertension (IH). If easy access to ophthalmological care is not provided, many patients may not present to an ophthalmologist until IH has irreversibly altered their vision. As presented in a case report by Medert et al., the initial detection of idiopathic intracranial hypertensionassociated papilledema in a male was made with the use of a comprehensive teleophthalmology screening examination, probably much earlier than it would have been if he had sought medical care in person [90]. An Italian study that scrutinized if smartphone-based ocular fundus photography could be used for the Emergency Department (ED) for hypertensive emergencies and compared it with traditional retinal 
fundoscopy, showed the decreased duration of examination and superior reliability of the smartphone-based method. This way, many missed diagnoses of acute hypertension emergencies can be avoided, and they can be conducted faster [91].

Coverage gaps in emergency ophthalmic care particularly in rural communities are a problematic situation that complicates and sets burdens for ophthalmic care delivery. This problem has been investigated widely in the USA, where the EDs are usually inaccessible or without the proper specialists and equipment for ophthalmic emergencies. In these studies, teleophthalmology is considered of high interest and perceived value for remote triage and consultations for both patients and health professionals [92,93].

The COVID-19 pandemic has resulted in altered hospital environments, with decreased outpatient activity and patients unwilling to visit the hospital. This situation forced the development of teleophthalmology so that emergencies are timely referred to and continuously managed. A video consultation platform "Attend Anywhere” in Moorfields Eye Hospital's accident and ED (London, UK) had thriving results for patient satisfaction, consultation duration and waiting time [94]. Additionally, in India, Murthy et al. demonstrated the use of teleophthalmology for acute ocular pain in the times of the COVID-19 pandemic, in order to decrease unnecessary hospital visits and provide guidelines to the practicing ophthalmologists for teleconsultation [95]. Teleophthalmology is now recognized as a tool not only for the pandemic era but also for facilitating ophthalmic care in general.

\section{Neuro-ophthalmology}

For neuro-ophthalmology, a subspecialty that relies on clinical examinations, the COVID-19 era has been challenging. In a survey conducted by Moss et al. [96], out of 208 practicing neuro-ophthalmologists, the majority of whom increased the use of teleophthalmology due to COVID-19 restriction, characterized access, continuity and patient efficiency of care as benefits and data quality and inaccuracy of fundoscopy as barriers of teleophthalmology. Video telehealth was considered most helpful for cranial nerve palsies, migraine with aura and optic neuritis and not helpful for various optic neuropathies. Grossman et al. [97] referred to the future implications of teleophthalmology in neuro-ophthalmology, highlighting the use in education for practicing residents for video-based teaching, evaluation and distance barrier solving.

Video visits, phone visits, online portal communication, provider-to-provider Consultation (“E-consult”), home testing with a smartphone for color vision and tablet for perimetry are all implementations of teleophthalmology that have been investigated and are utilized in neuro-ophthalmology practices, without replacing but facilitating clinical practice [98].

\section{Challenges to overcome/limitations}

As with every novel technological innovation, teleophthalmology and its implementations do not come without limitations. The benefit of teleophthalmology can be enormous and the possibilities numerous, but barriers exist and need to be addressed by the scientific community. Unfamiliarity with teleophthalmology for both patients and health professionals, as presented in Liu et al., highlighted the need for extensive training and uniform referral standards [45]. A consensus on image gradeability is essential for accurate grading and agreement between the health professionals. Misconceptions about diabetic eye screening and logistical challenges for patients and difficulty in the referral process and the use of teleophthalmology for primary care providers were barriers identified through interviews in a qualitative study in a rural US primary care clinic [11].

Even though minimum technical knowledge and resources for sharing images are needed for virtual consultations, photographs and even video calls do not provide as much information as can be gained by slit-lamp examination and important tests, such as intraocular pressure measurement, are not possible. Furthermore, several other issues, such as informed consent of the patient, payment for the consultation, medico-legal liability for the treating doctor and prescription of medication, need further deliberation and policy formation.

On patients' perspectives on teleophthalmology survey including willingness to pay (WTP), the cost was cited as a concern for which education is required, particularly in underserved and low-resourced populations [99].

Teleophthalmology can also put additional pressure on healthcare personnel creating the phenomenon or workarounds that maximize the number of tasks for ophthalmologists and increase patient volume. This way, the implementation of teleophthalmology in everyday clinical practice has to be very careful and needs to be adequately adapted in order to achieve the best results (Table 1) [100]. Non-medical healthcare professionals (e.g. trained optometrists, specialist nurses or specialist ophthalmic photographers) could assist with the grading of virtually obtained images and decrease the burden on the limited pool of medically trained staff. 


\section{Cureus}

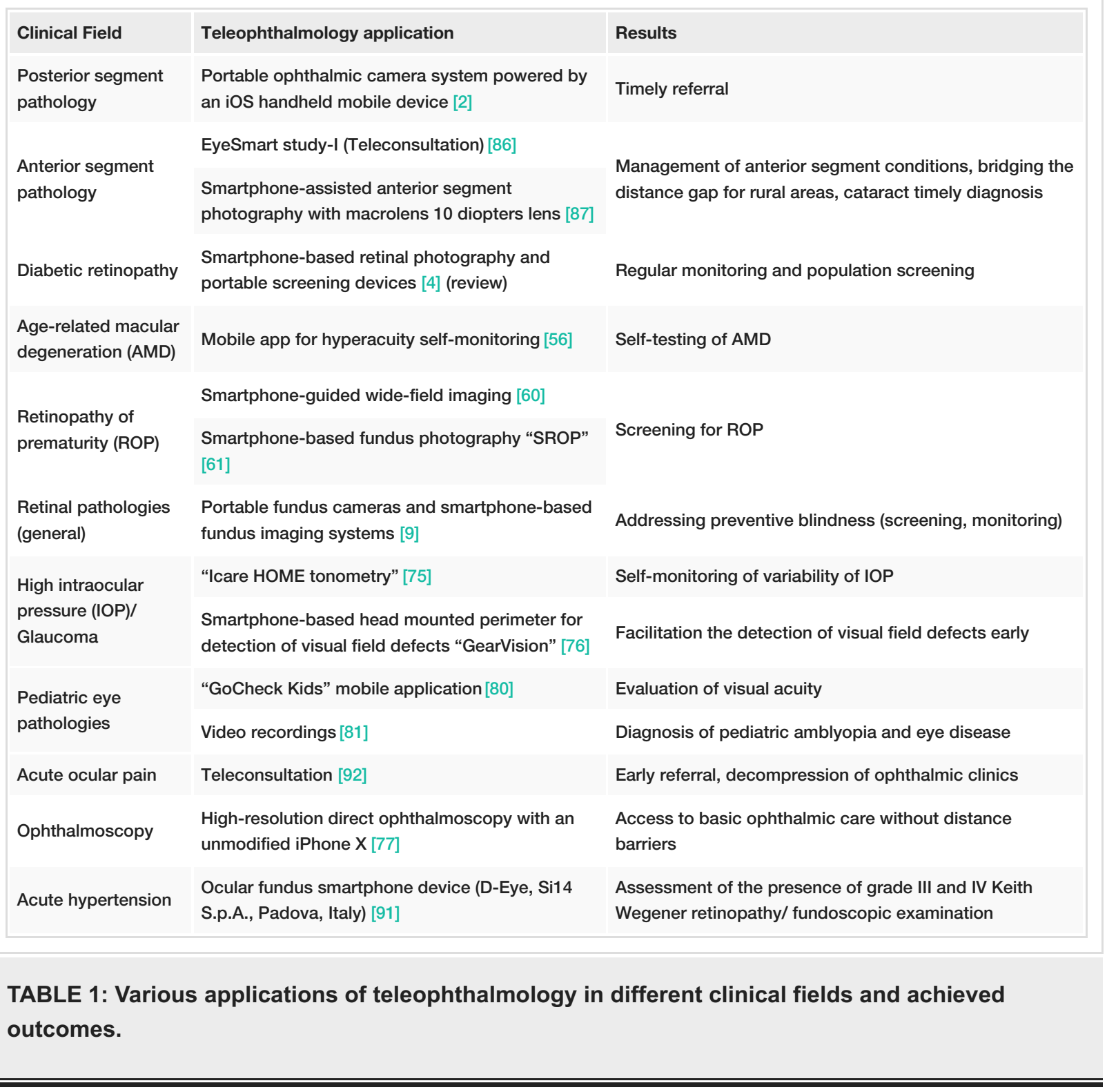

Artifacts can also appear when using fundus photography means for ophthalmology, and even if they are not much documented, they need to be addressed, possibly with optimization of imaging devices so that resulting in false-positive referral is avoided [101].

\section{Future perspectives}

The futures perspectives of teleophthalmology are broad and complex. The role of telemedicine in healthcare is expanding and the COVID-19 pandemic has accelerated the implementation of telemedical tools, especially in ophthalmology. Ophthalmologists are already embracing the novel technology while observing its benefits through the pandemic. Successful devices' use and programs implementation require carefulness and detailed research to overcome barriers concerning the cost-effectiveness and the ethical concerns for the acquired data [5,102].

Demographic and market requirements will also play an important role in telemedicine applications acting as guidance markers for additional research. Innovation, robust programs and improvement of existing devices are supposed to positively impact VA outcomes in visual-threatening diseases like DR and ROP. Future teleophthalmology could support more personalized management through continuous data recording $[103,104]$.

This data can be used for AI programs that come along with the development of teleophthalmology (Table 2). The combination of AI and teleophthalmology is likely to be revolutionary for ophthalmology when it is accurately integrated into ophthalmological practice $[105,106]$. 


\section{Cureus}

\section{Use}

Diabetic retinopathy screening

Diagnosis of plus and pre plus disease in an independent test set of 100 retinal images

Effective triage of the patients and facilitation of glaucoma clinical practice (not yet approved)

Assessment and diagnosis of adult and pediatric cataracts of varying severities

\section{Al system}

Software based on deep learning techniques [31]

Algorithm based on deep learning to automatically diagnose plus disease from retinal photographs $[65,66]$

Al with deep learning algorithm [78]

Deep learning algorithm, "ResNet" [89]

TABLE 2: Applications of artificial intelligence in ophthalmology.

Al - artificial intelligence

\section{Conclusions}

As the COVID-19 pandemic is still ongoing, the need for change and adaption of medical practice to the new circumstances is undoubtable. Teleophthalmology addresses several eye pathologies that otherwise could be overlooked, late diagnosed or incorrectly managed. Teleophthalmological devices and programs are being implemented and researched widely and have been demonstrating positive results. With the contribution of AI to teleophthalmological approaches studies are showing that this could be the future for ophthalmological practice.

Performance validation, safe data acquisition and economic feasibility constitute the main concerns for the implementation of teleophthalmological tools, while the benefits are steadily being recognized. Retinopathies, glaucoma, and age-related macular degeneration are being in the spotlight of teleophthalmological research so that visual impairment is prevented. Eye care delivery systems offering high-quality patient care will have to consider the movement of ophthalmological care from the doctor's office to the patients' homes. Toward the goal of providing the best possible ophthalmological care and reducing preventable visual impairment of any form, further studies need to be constructed and funded.

\section{Additional Information}

\section{Disclosures}

Conflicts of interest: In compliance with the ICMJE uniform disclosure form, all authors declare the following: Payment/services info: All authors have declared that no financial support was received from any organization for the submitted work. Financial relationships: All authors have declared that they have no financial relationships at present or within the previous three years with any organizations that might have an interest in the submitted work. Other relationships: All authors have declared that there are no other relationships or activities that could appear to have influenced the submitted work.

\section{References}

1. Kalavar M, Hua HU, Sridhar J: Teleophthalmology: an essential tool in the era of the novel coronavirus 2019 . Curr Opin Ophthalmol. 2020, 31:366-73. 10.1097/ICU.0000000000000689

2. Hong K, Collon S, Chang D, et al.: Teleophthalmology through handheld mobile devices: a pilot study in rural Nepal. J Mob Technol Med. 2019, 8: 10.7309/jmtm.8.1.1

3. Korot E, Wood E, Weiner A, Sim DA, Trese M: A renaissance of teleophthalmology through artificial intelligence. Eye (Lond). 2019, 33:861-3. 10.1038/s41433-018-0324-8

4. DeBuc DC: The role of retinal imaging and portable screening devices in tele-ophthalmology applications for diabetic retinopathy management. Curr Diab Rep. 2016, 16:132. 10.1007/s11892-016-0827-2

5. Saleem SM, Pasquale LR, Sidoti PA, Tsai JC: Virtual ophthalmology: telemedicine in a COVID-19 era . Am J Ophthalmol. 2020, 216:237-42. 10.1016/j.ajo.2020.04.029

6. Ghazala FR, Hamilton R, Giardini ME, Livingstone IAT: Teleophthalmology techniques increase ophthalmic examination distance. Eye (Lond). 2021, 35:1780-1. 10.1038/s41433-020-1085-8

7. Kern C, Fu DJ, Kortuem K, et al.: Implementation of a cloud-based referral platform in ophthalmology: making telemedicine services a reality in eye care. Br J Ophthalmol. 2020, 104:312-7. 10.1136/bjophthalmol2019-314161

8. Li JO, Liu H, Ting DSJ, et al.: Digital technology, tele-medicine and artificial intelligence in ophthalmology: a global perspective. Prog Retin Eye Res. 2021, 82:100900. 10.1016/j.preteyeres.2020.100900

9. Panwar N, Huang P, Lee J, et al.: Fundus photography in the 21 st century--a review of recent technological advances and their implications for worldwide healthcare. Telemed J E Health. 2016, 22:198-208. 10.1089/tmj.2015.0068

10. Chen N, Hsieh HP, Tsai RK, Sheu MM: Eye care services for the populations of remote districts in eastern 
Taiwan: a practical framework using a Mobile Vision Van Unit. Rural Remote Health. 2015, 15:3442.

11. Liu Y, Zupan NJ, Swearingen R, et al.: Identification of barriers, facilitators and system-based implementation strategies to increase teleophthalmology use for diabetic eye screening in a rural US primary care clinic: a qualitative study. BMJ Open. 2019, 9:e022594. 10.1136/bmjopen-2018-022594

12. Das T, Pappuru RR: Telemedicine in diabetic retinopathy: access to rural India . Indian J Ophthalmol. 2016, 64:84-6. 10.4103/0301-4738.178151

13. Das AV, Mididoddi S, Kammari P, Deepthi Davara N, Loomba A, Khanna R, Taneja M: App-based tele ophthalmology: a novel method of rural eye care delivery connecting tertiary eye care center and vision venters in India. Int J Telemed Appl. 2019, 2019:8107064. 10.1155/2019/8107064

14. Loomba A, Vempati S, Davara N, Shravani M, Kammari P, Taneja M, Das AV: Use of a tablet attachment in teleophthalmology for real-time video transmission from rural vision centers in a three-tier eye care network in India: eyeSmart cyclops. Int J Telemed Appl. 2019, 2019:5683085. 10.1155/2019/5683085

15. Cocozza VR, Santamaria JA, Giles GB, Keehn MC, Miller K: Teleophthalmology prevents unnecessary evacuation from warzone. Mil Med. 2020, 185:e909-11. 10.1093/milmed/usz329

16. Tan IJ, Dobson LP, Bartnik S, Muir J, Turner AW: Real-time teleophthalmology versus face-to-face consultation: a systematic review. J Telemed Telecare. 2017, 23:629-38. 10.1177/1357633X16660640

17. Collon S, Chang D, Tabin G, Hong K, Myung D, Thapa S: Utility and feasibility of teleophthalmology using a smartphone-based ophthalmic camera in screening camps in Nepal. Asia Pac J Ophthalmol (Phila). 2020, 9:54-8. 10.1097/01.APO.0000617936.16124.ba

18. Sommer AC, Blumenthal EZ: Telemedicine in ophthalmology in view of the emerging COVID-19 outbreak . Graefes Arch Clin Exp Ophthalmol. 2020, 258:2341-52. 10.1007/s00417-020-04879-2

19. Sharma M, Jain N, Ranganathan S, Sharma N, Honavar SG, Sharma N, Sachdev MS: Tele-ophthalmology: need of the hour. Indian J Ophthalmol. 2020, 68:1328-38. 10.4103/ijo.IJO_1784_20

20. De Fauw J, Ledsam JR, Romera-Paredes B, et al.: Clinically applicable deep learning for diagnosis and referral in retinal disease. Nat Med. 2018, 24:1342-50. 10.1038/s41591-018-0107-6

21. Chee RI, Darwish D, Fernandez-Vega A, et al.: Retinal telemedicine. Curr Ophthalmol Rep. 2018, 6:36-45. 10.1007/s40135-018-0161-8

22. Tufail A, Kapetanakis VV, Salas-Vega S, et al.: An observational study to assess if automated diabetic retinopathy image assessment software can replace one or more steps of manual imaging grading and to determine their cost-effectiveness. Health Technol Assess. 2016, 20:1-72. 10.3310/hta20920

23. El-Khayat AR, Anzidei R, Konidaris V: Ophthalmic photographer virtual clinics in medical retina. Int J Ophthalmol. 2020, 13:677-80. 10.18240/ijo.2020.04.23

24. Pathipati AS, Moshfeghi DM: Telemedicine applications in pediatric retinal disease . J Clin Med. 2017, 6:36. $10.3390 / j \mathrm{~cm} 6040036$

25. Chasan JE, Delaune B, Maa AY, Lynch MG: Effect of a teleretinal screening program on eye care use and resources. JAMA Ophthalmol. 2014, 132:1045-51. 10.1001/jamaophthalmol.2014.1051

26. Kozak I, Payne JF, Schatz P, Al-Kahtani E, Winkler M: Teleophthalmology image-based navigated retinal laser therapy for diabetic macular edema: a concept of retinal telephotocoagulation. Graefes Arch Clin Exp Ophthalmol. 2017, 255:1509-13. 10.1007/s00417-017-3674-1

27. Lee R, Wong TY, Sabanayagam C: Epidemiology of diabetic retinopathy, diabetic macular edema and related vision loss. Eye Vis (Lond). 2015, 2:17. 10.1186/s40662-015-0026-2

28. Yau JW, Rogers SL, Kawasaki R, et al.: Global prevalence and major risk factors of diabetic retinopathy . Diabetes Care. 2012, 35:556-64. 10.2337/dc11-1909

29. Bilong Y, Katte JC, Koki G, et al.: Validation of smartphone-based retinal photography for diabetic retinopathy screening. Ophthalmic Surg Lasers Imaging Retina. 2019, 50:S18-22. 10.3928/2325816020190108-05

30. Wong TY, Bressler NM: Artificial intelligence with deep learning technology looks into diabetic retinopathy screening. JAMA. 2016, 316:2366-7. 10.1001/jama.2016.17563

31. Gulshan V, Peng L, Coram M, et al.: Development and validation of a deep learning algorithm for detection of diabetic retinopathy in retinal fundus photographs. JAMA. 2016, 316:2402-10. 10.1001/jama.2016.17216

32. Arcadu F, Benmansour F, Maunz A, et al.: Deep learning predicts OCT measures of diabetic macular thickening from color fundus photographs. Invest Ophthalmol Vis Sci. 2019, 60:852-7. 10.1167/iovs.1825634

33. Zimmer-Galler IE, Kimura AE, Gupta S: Diabetic retinopathy screening and the use of telemedicine . Curr Opin Ophthalmol. 2015, 26:167-72. 10.1097/ICU.0000000000000142

34. Tozer K, Woodward MA, Newman-Casey PA: Telemedicine and diabetic retinopathy: review of published screening programs. J Endocrinol Diabetes. 2015, 2: 10.15226/2374-6890/2/4/00131

35. Rodríguez Villa S, Alonso Álvarez C, de Dios Del Valle R, et al.: Five-year experience of tele-ophthalmology for diabetic retinopathy screening in a rural population. Arch Soc Esp Oftalmol. 2016, 91:426-30. 10.1016/j.oftal.2016.01.023

36. Boucher MC, El Yamani MEM: Urban diabetic retinopathy teleophthalmology screening: results and impact at the service corridor. Can J Ophthalmol. 2019, 54:359-66. 10.1016/j.jcjo.2018.06.008

37. Vujosevic S, Pucci P, Casciano M, et al.: A decade-long telemedicine screening program for diabetic retinopathy in the north-east of Italy. J Diabetes Complications. 2017, 31:1348-53. 10.1016/j.jdiacomp.2017.04.010

38. Invernizzi A, Bevilacqua MT, Cozzi M, Bianchi C, Pagani A, Cigada M, Staurenghi G: Diabetic retinopathy screening: the first telemedical approach in an Italian hospital. Eur J Ophthalmol. 2016, 26:369-74. 10.5301/ejo.5000719

39. Sim DA, Mitry D, Alexander P, et al.: The evolution of teleophthalmology programs in the United Kingdom: beyond diabetic retinopathy screening. J Diabetes Sci Technol. 2016, 10:308-17. 10.1177/1932296816629983

40. Kanjee R, Dookeran RI: Tele-ophthalmology for diabetic retinopathy in Canada-meeting the needs of a growing epidemic. Can J Ophthalmol. 2017, 52 Suppl 1:S13-4. 10.1016/j.jcjo.2017.09.021

41. Matimba A, Woodward R, Tambo E, Ramsay M, Gwanzura L, Guramatunhu S: Tele-ophthalmology: opportunities for improving diabetes eye care in resource- and specialist-limited Sub-Saharan African 
countries. J Telemed Telecare. 2016, 22:311-6. 10.1177/1357633X15604083

42. Liu Y, Torres Diaz A, Benkert R: Scaling up teleophthalmology for diabetic eye screening: opportunities for widespread implementation in the USA. Curr Diab Rep. 2019, 19:74. 10.1007/s11892-019-1187-5

43. Avendaño-Veloso A, Parada-Hernández F, González-Ramos R, Dougnac-Osses C, Carrasco-Sáez JL, Scanlon PH: Teleophthalmology: a strategy for timely diagnosis of sight-threatening diabetic retinopathy in primary care, Concepción, Chile. Int J Ophthalmol. 2019, 12:1474-8. 10.18240/ijo.2019.09.16

44. Silva PS, Horton MB, Clary D, Lewis DG, Sun JK, Cavallerano JD, Aiello LP: Identification of diabetic retinopathy and ungradable image rate with ultrawide field imaging in a national teleophthalmology program. Ophthalmology. 2016, 123:1360-7. 10.1016/j.ophtha.2016.01.043

45. Liu Y, Rajamanickam VP, Parikh RS, et al.: Diabetic retinopathy assessment variability among eye care providers in an urban teleophthalmology program. Telemed J E Health. 2019, 25:301-8. 10.1089/tmj.2018.0019

46. Silva PS, Cavallerano JD, Tolson AM, et al.: Real-time ultrawide field image evaluation of retinopathy in a diabetes telemedicine program. Diabetes Care. 2015, 38:1643-9. 10.2337/dc15-0161

47. Coronado AC, Zaric GS, Martin J, Malvankar-Mehta M, Si FF, Hodge WG: Diabetic retinopathy screening with pharmacy-based teleophthalmology in a semiurban setting: a cost-effectiveness analysis. CMAJ Open. 2016, 4:E95-102. 10.9778/cmajo.20150085

48. Boucher MC, Ouazani Chahdi H, El Yamani MEM: Compliance to follow-up care after urban diabetic retinopathy tele-screening. Can J Ophthalmol. 2020, 55:2-7. 10.1016/j.jcjo.2019.01.001

49. Li JQ, Welchowski T, Schmid M, Mauschitz MM, Holz FG, Finger RP: Prevalence and incidence of age-related macular degeneration in Europe: a systematic review and meta-analysis. Br J Ophthalmol. 2020, 104:107784. 10.1136/bjophthalmol-2019-314422

50. Ambati J, Ambati BK, Yoo SH, Ianchulev S, Adamis AP: Age-related macular degeneration: etiology, pathogenesis, and therapeutic strategies. Surv Ophthalmol. 2003, 48:257-93. 10.1016/s0039-6257(03)000304

51. Brady CJ, Garg S: Telemedicine for age-related macular degeneration. Telemed J E Health. 2020, 26:565-8. 10.1089/tmj.2020.0011

52. Kawaguchi A, Sharafeldin N, Sundaram A, et al.: Tele-ophthalmology for age-related macular degeneration and diabetic retinopathy screening: a systematic review and meta-analysis. Telemed J E Health. 2018, 24:301-8. 10.1089/tmj.2017.0100

53. Nanji K, Kherani IN, Damji KF, Nyenze M, Kiage D, Tennant MT: The Muranga teleophthalmology study: a comparison of virtual (Teleretina) assessment with in-person clinical examination to diagnose diabetic retinopathy and age-related macular degeneration in Kenya. Middle East Afr J Ophthalmol. 2020, 27:91-9. 10.4103/meajo.MEAJO_144_19

54. Li B, Powell AM, Hooper PL, Sheidow TG: Prospective evaluation of teleophthalmology in screening and recurrence monitoring of neovascular age-related macular degeneration: a randomized clinical trial. JAMA Ophthalmol. 2015, 133:276-82. 10.1001/jamaophthalmol.2014.5014

55. Tsaousis KT, Empeslidis T, Konidaris VE, Kapoor B, Deane J: The concept of virtual clinics in monitoring patients with age-related macular degeneration. Acta Ophthalmol. 2016, 94:e353-5. 10.1111/aos.12832

56. Schmid MK, Thiel MA, Lienhard K, Schlingemann RO, Faes L, Bachmann LM: Reliability and diagnostic performance of a novel mobile app for hyperacuity self-monitoring in patients with age-related macular degeneration. Eye (Lond). 2019, 33:1584-9. 10.1038/s41433-019-0455-6

57. Blencowe H, Lawn JE, Vazquez T, Fielder A, Gilbert C: Preterm-associated visual impairment and estimates of retinopathy of prematurity at regional and global levels for 2010. Pediatr Res. 2013, 74 Suppl 1:35-49. 10.1038/pr.2013.205

58. Gilbert C: Retinopathy of prematurity: a global perspective of the epidemics, population of babies at risk and implications for control. Early Hum Dev. 2008, 84:77-82. 10.1016/j.earlhumdev.2007.11.009

59. Biten H, Redd TK, Moleta C, et al.: Diagnostic accuracy of ophthalmoscopy vs telemedicine in examinations for retinopathy of prematurity. JAMA Ophthalmol. 2018, 136:498-504. 10.1001/jamaophthalmol.2018.0649

60. Patel TP, Aaberg MT, Paulus YM, et al.: Smartphone-based fundus photography for screening of plusdisease retinopathy of prematurity. Graefes Arch Clin Exp Ophthalmol. 2019, 257:2579-85. 10.1007/s00417019-04470-4

61. Goyal A, Gopalakrishnan M, Anantharaman G, Chandrashekharan DP, Thachil T, Sharma A: Smartphone guided wide-field imaging for retinopathy of prematurity in neonatal intensive care unit - a Smart ROP (SROP) initiative. Indian J Ophthalmol. 2019, 67:840-5. 10.4103/ijo.IJO_1177_18

62. Mora JS, Waite C, Gilbert CE, Breidenstein B, Sloper JJ: A worldwide survey of retinopathy of prematurity screening. Br J Ophthalmol. 2018, 102:9-13. 10.1136/bjophthalmol-2017-310709

63. Wallace DK: Fellowship training in retinopathy of prematurity . J AAPOS. 2012, 16:1. 10.1016/j.jaapos.2011.11.003

64. Brady CJ, D'Amico S, Campbell JP: Telemedicine for retinopathy of prematurity. Telemed J E Health. 2020, 26:556-64. 10.1089/tmj.2020.0010

65. Brown JM, Campbell JP, Beers A, et al.: Automated diagnosis of plus disease in retinopathy of prematurity using deep convolutional neural networks. JAMA Ophthalmol. 2018, 136:803-10. 10.1001/jamaophthalmol.2018.1934

66. Scruggs BA, Chan RVP, Kalpathy-Cramer J, Chiang MF, Campbell JP: Artificial intelligence in retinopathy of prematurity diagnosis. Transl Vis Sci Technol. 2020, 9:5. 10.1167/tvst.9.2.5

67. Hu J, Chen Y, Zhong J, Ju R, Yi Z: Automated analysis for retinopathy of prematurity by deep neural networks. IEEE Trans Med Imaging. 2019, 38:269-79. 10.1109/TMI.2018.2863562

68. Gan K, Liu Y, Stagg B, Rathi S, Pasquale LR, Damji K: Telemedicine for glaucoma: guidelines and recommendations. Telemed J E Health. 2020, 26:551-5. 10.1089/tmj.2020.0009

69. Quigley HA, Broman AT: The number of people with glaucoma worldwide in 2010 and 2020 . Br J Ophthalmol. 2006, 90:262-7. 10.1136/bjo.2005.081224

70. Delgado MF, Abdelrahman AM, Terahi M, et al.: Management of glaucoma In developing countries: challenges and opportunities for improvement. Clinicoecon Outcomes Res. 2019, 11:591-604. 


\subsection{7/CEOR.S218277}

71. Choudhari NS, Chandran P, Rao HL, Jonnadula GB, Addepalli UK, Senthil S, Garudadri CS: LVPEI glaucoma epidemiology and molecular genetic study: teleophthalmology screening for angle-closure disease in an underserved region. Eye (Lond). 2020, 34:1399-405. 10.1038/s41433-019-0666-X

72. Modjtahedi BS, Chu K, Luong TQ, et al.: Two-year outcomes of a pilot glaucoma suspect telemedicine monitoring program. Clin Ophthalmol. 2018, 12:2095-102. 10.2147/OPTH.S171263

73. Odden JL, Khanna CL, Choo CM, et al.: Telemedicine in long-term care of glaucoma patients. J Telemed Telecare. 2020, 26:92-9. 10.1177/1357633X18797175

74. Mursch-Edlmayr AS, Ng WS, Diniz-Filho A, et al.: Artificial intelligence algorithms to diagnose glaucoma and detect glaucoma progression: translation to clinical practice. Transl Vis Sci Technol. 2020, 9:55. 10.1167/tvst.9.2.55

75. Cvenkel B, Atanasovska Velkovska M: Self-monitoring of intraocular pressure using Icare HOME tonometry in clinical practice. Clin Ophthalmol. 2019, 13:841-7. 10.2147/OPTH.S198846

76. Sircar T, Mishra A, Bopardikar A, Tiwari VN: GearVision: smartphone based head mounted perimeter for dtection of visual field defects. Annu Int Conf IEEE Eng Med Biol Soc. 2018, 2018:5402-5. 10.1109/EMBC.2018.8513574

77. Gunasekera CD, Thomas P: High-resolution direct ophthalmoscopy with an unmodified iPhone X. JAMA Ophthalmol. 2019, 137:212-3. 10.1001/jamaophthalmol.2018.5806

78. Mayro EL, Wang M, Elze T, Pasquale LR: The impact of artificial intelligence in the diagnosis and management of glaucoma. Eye (Lond). 2020, 34:1-11. 10.1038/s41433-019-0577-X

79. Jain M, Rojanaporn D, Chawla B, Sundar G, Gopal L, Khetan V: Retinoblastoma in Asia. Eye (Lond). 2019, 33:87-96. 10.1038/s41433-018-0244-7

80. Silverstein E, Williams JS, Brown JR, Bylykbashi E, Stinnett SS: Teleophthalmology: evaluation of phonebased visual acuity in a pediatric population. Am J Ophthalmol. 2021, 221:199-206. 10.1016/j.ajo.2020.08.007

81. Sabri K, Moinul P, Tehrani N, Wiggins R, Fleming N, Farrokhyar F: Video interpretation and diagnosis of pediatric amblyopia and eye disease. J Telemed Telecare. 2021, 27:116-22. 10.1177/1357633X19864823

82. Young K, Gupta A, Palacios R: Impact of telemedicine in pediatric postoperative care. Telemed J E Health. 2019, 25:1083-9. 10.1089/tmj.2018.0246

83. Reid JE, Eaton E: Artificial intelligence for pediatric ophthalmology. Curr Opin Ophthalmol. 2019, 30:33746. 10.1097/ICU.0000000000000593

84. Hu WF, Lorch AC: Teleophthalmology for anterior segment disease . Int Ophthalmol Clin. 2019, 59:55-67. 10.1097/IIO.0000000000000287

85. Ting DSJ, Foo VH, Yang LWY, et al.: Artificial intelligence for anterior segment diseases: emerging applications in ophthalmology. Br J Ophthalmol. 2021, 105:158-68. 10.1136/bjophthalmol-2019-315651

86. Misra N, Khanna RC, Mettla AL, Marmamula S, Rathi VM, Das AV: Role of teleophthalmology to manage anterior segment conditions in vision centres of south India: EyeSmart study-I. Indian J Ophthalmol. 2020, 68:362-7. 10.4103/ijo.IJO_991_19

87. Mohammadpour M, Mohammadpour L, Hassanzad M: Smartphone assisted slit lamp free anterior segment imaging: a novel technique in teleophthalmology. Cont Lens Anterior Eye. 2016, 39:80-1. 10.1016/j.clae.2015.09.005

88. Ting DSJ, Ang M, Mehta JS, Ting DSW: Artificial intelligence-assisted telemedicine platform for cataract screening and management: a potential model of care for global eye health. Br J Ophthalmol. 2019, 103:1537-8. 10.1136/bjophthalmol-2019-315025

89. Wu X, Huang Y, Liu Z, et al.: Universal artificial intelligence platform for collaborative management of cataracts. Br J Ophthalmol. 2019, 103:1553-60. 10.1136/bjophthalmol-2019-314729

90. Medert CM, Lynch MG, Maa AY: Detection of idiopathic intracranial hypertension, enabled by teleophthalmology. Can J Ophthalmol. 2017, 52:e117-20. 10.1016/j.jcjo.2016.11.009

91. Muiesan ML, Salvetti M, Paini A, et al.: Ocular fundus photography with a smartphone device in acute hypertension. J Hypertens. 2017, 35:1660-5. 10.1097/HJH.0000000000001354

92. Tauber J, Ayoub S, Shah P, Wu M, Tsui E, Schuman JS, Rathi S: Assessing the demand for teleophthalmology in Florida Emergency Departments. Telemed J E Health. 2020, 26:1500-6. 10.1089/tmj.2019.0260

93. Wedekind L, Sainani K, Pershing S: Supply and perceived demand for teleophthalmology in triage and consultations in California Emergency Departments. JAMA Ophthalmol. 2016, 134:537-43. 10.1001/jamaophthalmol.2016.0316

94. Kilduff CL, Thomas AA, Dugdill J, et al.: Creating the Moorfields' virtual eye casualty: video consultations to provide emergency teleophthalmology care during and beyond the COVID-19 pandemic. BMJ Health Care Inform. 2020, 27:e100179. 10.1136/bmjhci-2020-100179

95. Murthy SI, Das S, Deshpande P, et al.: Differential diagnosis of acute ocular pain: teleophthalmology during COVID-19 pandemic - a perspective. Indian J Ophthalmol. 2020, 68:1371-9. 10.4103/ijo.IJO_1267_20

96. Moss HE, Lai KE, Ko MW: Survey of telehealth adoption by neuro-ophthalmologists during the COVID-19 pandemic: benefits, barriers, and utility. J Neuroophthalmol. 2020, 40:346-55. 10.1097/WNO.0000000000001051

97. Grossman SN, Calix R, Tow S, et al.: Neuro-ophthalmology in the era of COVID-19: future implications of a public health crisis. Ophthalmology. 2020, 127:e72-4. 10.1016/j.ophtha.2020.05.004

98. Lai KE, Ko MW, Rucker JC, et al.: Tele-neuro-ophthalmology during the age of COVID-19. J Neuroophthalmol. 2020, 40:292-304. 10.1097/WNO.0000000000001024

99. Ramchandran RS, Yilmaz S, Greaux E, Dozier A: Patient perceived value of teleophthalmology in an urban, low income US population with diabetes. PLoS One. 2020, 15 :e0225300. 10.1371/journal.pone.0225300

100. Bouskill K, Smith-Morris C, Bresnick G, Cuadros J, Pedersen ER: Blind spots in telemedicine: a qualitative study of staff workarounds to resolve gaps in diabetes management. BMC Health Serv Res. 2018, 18:617. 10.1186/s12913-018-3427-9

101. Ranjan R, Shah PK, Narendran V: Curious case of retrolental mass: an unusual tele-screening artifact . Indian J Ophthalmol. 2019, 67:415-6. 10.4103/ijo.IJO_1318_18 


\section{Cureus}

102. Bashshur R, Doarn CR, Frenk JM, Kvedar JC, Woolliscroft JO: Telemedicine and the COVID-19 pandemic, lessons for the future. Telemed J E Health. 2020, 26:571-3. 10.1089/tmj.2020.29040.rb

103. Holekamp NM: Moving from clinic to home: what the future holds for ophthalmic telemedicine . Am J Ophthalmol. 2018, 187:xxviii-xv. 10.1016/j.ajo.2017.11.003

104. Parikh D, Armstrong G, Liou V, Husain D: Advances in telemedicine in ophthalmology. Semin Ophthalmol. 2020, 35:210-5. 10.1080/08820538.2020.1789675

105. Kapoor R, Walters SP, Al-Aswad LA: The current state of artificial intelligence in ophthalmology . Surv Ophthalmol. 2019, 64:233-40. 10.1016/j.survophthal.2018.09.002

106. Hogarty DT, Mackey DA, Hewitt AW: Current state and future prospects of artificial intelligence in ophthalmology: a review. Clin Exp Ophthalmol. 2019, 47:128-39. 10.1111/ceo.13381 